\title{
NEUTRON STARQUAKE MODELS FOR GAMMA-RAY BURSTS
}

\author{
O. Blaes, R. Blandford, P. Goldreich, and P. Madau \\ Theoretical Astrophysics, California Institute of Technology, Pasadena \\ Received 1988 August 8; accepted 1989 January 20
}

\begin{abstract}
We assess neutron starquake models for $\gamma$-ray bursts. The elastic energy the crust can store is sufficient to account for that radiated in a single burst, but it is insufficient to supply the $\gtrsim 10^{6}$ bursts each star produces over its lifetime, and so it must be replenished. Seismic waves are radiated if shear stress is relieved by brittle fracture. However they cannot propagate directly to the surface but are temporarily trapped below a reflecting layer. Between the reflecting layer and the surface the displacement amplitude of the wave is nearly constant and the strain is very small. At low frequencies, $\lesssim 10^{4} \mathrm{~Hz}$, the reflection is associated with an evanescent zone. At high frequencies, $\gtrsim 10^{4} \mathrm{~Hz}$, the reflection occurs where the magnetic field stress starts to dominate the crustal rigidity. The shaking of the stellar surface couples the seismic waves to Alfvén waves which propagate out into the magnetosphere. At low frequencies, the coupling coefficient, $T$, is proportional to the square of the magnetic field, $B$, and increases with the seventh power of the wave frequency, $v$. At high frequencies, $T$ is proportional to $B^{4 / 7} v^{3 / 7}$. Alfvén wave luminosities sufficient to power Galactic $\gamma$-ray bursts are possible if magnetic fields $\gtrsim 10^{11} \mathrm{G}$ cover at least part of the stellar surface. The conversion of Alfvén waves into $\gamma$-rays may occur if the waves are charge-starved or if their amplitudes approach that of the background magnetic field.

Subject headings: gamma rays: bursts - stars: neutron - wave motions
\end{abstract}

\section{INTRODUCTION}

Fifteen years after their discovery by Klebesadel, Strong and Olson (1973), $\gamma$-ray bursts remain a major unsolved problem of high-energy astrophysics. Satellites detect around a hundred bursts per year. The bursts lack counterparts at other wavelengths (e.g., Hartmann and Woosley 1988), and consequently the source objects are unidentified. Individual bursts usually last for a few seconds; they exhibit a great variety of temporal structure, including rise times as short as several milliseconds (e.g., Liang and Petrosian 1986). Observed fluences lie in the range $10^{-6}$ to $10^{-4} \mathrm{ergs} \mathrm{cm}^{-2}$, with substantial emission above a few $\mathrm{MeV}$ being common (Share et al. 1986). The narrow distribution of fluences suggests that the luminosity function is not very broad.

Neutron stars have long been viewed as being the most plausible sources. The positional coincidence of the peculiar 1979 March 5 event with a supernova remnant in the LMC and its fast $(<0.25 \mathrm{~ms})$ rise time and $8 \mathrm{~s}$ pulsations provide the strongest links to rotating neutron stars. The recently discovered $2.2 \mathrm{~s}$ periodicity in the 1984 August 5 event (Kouveliotou et al. 1988) is also suggestive. Other circumstantial (and controversial) evidence pointing to neutron stars includes linelike features at $\sim 50$ and $\sim 450 \mathrm{keV}$, respectively interpreted as due to cyclotron resonance in $\mathrm{a} \sim 10^{12} \mathrm{G}$ magnetic field and gravitationally redshifted $\gamma$-rays from $e^{ \pm}$pair annihilation (e.g., Liang and Petrosian 1986).

The distribution of bursts over the sky is isotropic, and so they must be located within the neutron star scale height, $\sim 1$ kpc. For many events, source distances less than a kiloparsec are also inferred from the absence of a spectral cutoff (due to photon-photon pair production) above a few $\mathrm{MeV}$ (Schmidt 1978).

The relative lack of low-energy $\mathrm{X}$-ray luminosity, $L_{x}(3-10$ $\mathrm{keV}) / L_{\gamma}(>30 \mathrm{keV}) \simeq 0.02$ (Laros et al. 1984), is an important clue to understanding the nature of the $\gamma$-ray emission region.
This $X$-ray paucity constraint (Imamura and Epstein 1987) is unlikely to be satisfied in regions of high density or near the neutron star surface, where substantial thermal reprocessing of $\gamma$-rays into X-rays would occur. For sources associated with neutron stars this suggests emission from a region of lowdensity plasma well out in the magnetosphere. This geometry would also avoid an excessive optical depth to pair production.

Starquake models for $\gamma$-ray bursts have been discussed by Pacini and Ruderman (1974); Tsygan (1975); Fabian, Icke, and Pringle (1976); Muslimov and Tsygan (1986); and Epstein (1988). The basic idea is quite simple. Elastic energy released in a crustquake excites oscillations of the magnetic field frozen in the surface, and the induced electric field accelerates highenergy particles, which in turn radiate $\gamma$-rays. This paper is devoted to assessing the merits of starquakes as the root cause of $\gamma$-ray bursts.

Before embarking on a discussion of the consequences of neutron starquakes, it is only fair to point out that their very existence is open to question. Even if mechanisms exist for producing the requisite stress in the crust, stress release might proceed by plastic flow rather than by brittle fracture, especially at the depths implied by the quake energetics discussed below. Deep-focus earthquakes provide a good analogy; it is still not understood how rupture can occur at their depths (Kasahara 1981).

The plan of this paper is to assess the component parts of $\gamma$-ray burst models based on neutron starquakes. Section II contains a discussion of both the requirements for, and the expected properties of, neutron starquakes. The coupling of seismic waves to Alfvén waves is evaluated in $\S$ III, and the behavior of Alfvén waves in the magnetosphere is investigated in $\S$ IV. In $\S \mathrm{V}$ we attempt to identify the principal sources of free energy in the interiors of old neutron stars. Finally, $\S$ VI presents a brief overview of the strengths and weakness of starquake models for $\gamma$-ray bursts. 


\section{QUAKE ENERGETICS}

The energy released in a typical $\gamma$-ray burst, assuming isotopic emission, is

$$
E \approx \frac{10^{38}}{\eta}\left(\frac{F}{10^{-6} \mathrm{ergs} \mathrm{cm}^{-2}}\right)\left(\frac{D}{1 \mathrm{kpc}}\right)^{2} \text { ergs },
$$

where $\eta$ specifies the fraction of energy released which is converted into $\gamma$-rays, $F$ is the observed fluence, and $D$ is the distance. The magnetic energy in the magnetosphere $\left(\sim 10^{41}\right.$ ergs for a $10^{12} \mathrm{G}$ surface field) and the maximum elastic energy that the crust can store $\left(\sim 10^{44}\right.$ ergs for a $10^{-2}$ yield strain) are much greater than the typical energy required for a single burst, provided that $\eta$ is not too small. It is therefore plausible that each burst is produced by a starquake.

To examine the energetics of a starquake, we model the crust as plane-parallel, chemically homogeneous, and subject to a constant Newtonian gravitational acceleration, $g=-g \hat{z}$, where $\hat{z}$ is a unit vector in the upward direction. In numerical expressions we take $g=10^{14} \mathrm{~cm} \mathrm{~s}^{-2}$. We are primarily concerned with the region above neutron drip, which occurs at $\rho \approx 4 \times 10^{11} \mathrm{~g}$. Here the pressure is due to degenerate electrons. Integrating the equation of hydrostatic equilibrium, we obtain the density profile

$$
\rho=\frac{\left(\mu_{e} m_{u}\right)^{5 / 2}}{3 \pi^{2} \hbar^{3}}\left(\frac{g^{2} \mu_{e} m_{u}}{c^{2}} z^{2}+2 g m_{e}|z|\right)^{3 / 2},
$$

where $m_{u}$ is the atomic mass unit, $\mu_{e}$ is the mean molecular weight per electron, and all other symbols have their usual meanings. We adopt an intermediate value of $\mu_{e}=2.5$ when presenting numerical results; detailed models predict that $\mu_{e}$ rises from about 2.2 near the surface to 3.3 near neutron drip (Baym, Pethick, and Sutherland 1971).

The ions in the solid crust are arranged in a Coulomb lattice whose shear modulus is given by (Baym and Pines 1971)

$$
\mu=0.295 Z^{2} e^{2} n_{i}^{4 / 3}
$$

where $n_{i}=\rho /\left(Z \mu_{e} m_{u}\right)$ is the ion number density. The atomic number, $Z$, varies from 26 near the surface to 36 near neutron drip (Baym, Pethick, and Sutherland 1971); an average value of 32 is adopted here. The crust will remain solid below the melting temperature

$$
T_{m}=\frac{Z^{5 / 3} e^{2}}{k \Gamma_{m}}\left(\frac{4 \pi \rho}{3 \mu_{e} m_{u}}\right)^{1 / 3} \sim 3 \times 10^{5} \rho^{1 / 3} \mathrm{~K},
$$

where $\Gamma_{m} \simeq 180$ is the melting parameter (Slattery, Doolen, and DeWitt 1982).

The elastic energy released in a quake may be expressed as

$$
E_{Q} \sim \mu\left(z_{Q}\right) \epsilon_{\text {yield }}^{2} d^{3},
$$

where $\epsilon_{\text {yield }}$ is the yield strain at which the crust cracks, $d^{2}$ is the area of the fault plane, and $z_{Q}$ is the depth. Equations (3) and (5) together require

$$
\begin{array}{r}
d \sim 3 \times 10^{4}\left(\frac{E_{Q}}{10^{38} \text { ergs }}\right)^{1 / 3}\left[\frac{\rho\left(z_{Q}\right)}{4 \times 10^{11} \mathrm{~g} \mathrm{~cm}^{-3}}\right]^{-4 / 9} \\
\quad \times\left(\frac{\epsilon_{\text {yield }}}{10^{-2}}\right)^{-2 / 3} \mathrm{~cm},
\end{array}
$$

where, for convenience, the density is scaled by its value at neutron drip.

The characteristic frequency, $v_{0}$, of elastic waves emitted by the quake is simply the speed at which the fracture propagates, divided by $d$. Since cracks typically propagate at a significant fraction of the shear wave speed, $v_{s}=(\mu / \rho)^{1 / 2}$, we find

$$
\begin{aligned}
v_{0} \sim 10^{4}\left(\frac{E_{Q}}{10^{38} \mathrm{ergs}}\right)^{-1 / 3} & {\left[\frac{\rho\left(z_{Q}\right)}{4 \times 10^{11} \mathrm{~g} \mathrm{~cm}^{-3}}\right]^{11 / 18} } \\
& \times\left(\frac{\epsilon_{\text {yield }}}{10^{-2}}\right)^{2 / 3} \mathrm{~Hz} .
\end{aligned}
$$

Simple models of fracture which successfully describe seismic radiation by terrestrial quakes (see, e.g., Kasahara 1981) predict that the spectral density, $E_{v}$, satisfies

$$
v E_{v} \propto \begin{cases}v^{3} & \text { for } v<v_{0}, \\ v^{-1} & \text { for } v>v_{0},\end{cases}
$$

so a substantial amount of energy is released at $v>v_{0}$. The seismic energy density is partitioned into shear and compressional waves in proportion to the inverse sixth power of the ratio of their propagation speeds. ${ }^{1}$ Most of the energy is emitted in shear waves, since their speed is typically a few times smaller than that of compressional waves. Some conversion of energy between shear and compressional waves occurs each time the waves are reflected below the stellar surface. We ignore the compressional waves in the remainder of the paper, since, for our purposes, their behavior is not very different from that of shear waves.

For simplicity we have assumed in this section that the quake occurs at or above the depth at which neutron drip occurs, and we see that this is sufficient to explain the burst energetics and time scales. More energy could be released from quakes at greater depths. However, the shear speed decreases with depth below neutron drip, and thus some of the waves produced in this region may never reach the surface.

\section{TRANSMISSION OF ENERGY INTO THE MAGNETOSPHERE}

The magnetic energy density dominates the rigidity in the outer layers of the crust of magnetic neutron stars. It may even exceed the rest-mass energy density very close to the surface, and it certainly does so in the magnetosphere. To include the effects of the magnetic field $\boldsymbol{B}$ on wave propagation, we add the Maxwell stress to the equations of elastodynamics. Because $B^{2} \gg 4 \pi \rho c^{2}$ in the magnetosphere, the displacement current cannot be neglected in Maxwell's equations. For simplicity, we consider the crust to have a plane-parallel geometry and the unperturbed magnetic field to be uniform. ${ }^{2}$ Moreover, we treat the surface layers as solid because, with our scalings, the magnetic field completely dominates the stress in the ocean provided that the surface temperature is $\lesssim 3 \times 10^{5} \mathrm{~K}$. This is probably true for old neutron stars.

Linearizing the equation of motion and the continuity equation about a static equilibrium, we obtain

$$
\rho \frac{\partial^{2} \xi}{\partial t^{2}}=\nabla \cdot \delta \sigma+\frac{1}{c} \delta j \times B+\delta \rho g-\nabla \delta p
$$

and

$$
\delta \rho=-\nabla \cdot(\rho \xi),
$$

\footnotetext{
${ }^{1}$ This dependence of energy density on wave speed is characteristic of a quadrupolar emission process. Because of the absence of external forces, quadrupoles are the lowest order multipoles in these seismic events.

2 The global structure of the magnetic field is important at frequencies $\left(\sim 5 \times 10^{3} \mathrm{~Hz}\right)$ below which the Alfvén wavelength is larger than the stellar radius.
} 
respectively. Here $\delta$ denotes an Eulerian perturbation, $\xi$ is the displacement of an element of material from its equilibrium position, and $\delta j$ is the perturbed current density. The pressure due to the degenerate electrons, $p$, has been written separately from the elastic stress associated with the deformation of the Coulomb lattice, $\sigma$. The components of the perturbed elastic stress tensor are related to the components of the gradient of $\xi$ by

$$
\delta \sigma_{i j}=\left(\kappa-\frac{2 \mu}{3}\right) \delta_{i j} \nabla \cdot \xi+\mu\left(\frac{\partial \xi_{i}}{\partial x_{j}}+\frac{\partial \xi_{j}}{\partial x_{i}}\right)
$$

where $\kappa$ is the bulk modulus. In writing equation (11), we have neglected the extra terms which arise if there is a static elastic stress associated with the equilibrium state. It is easy to show that these terms are of order $\epsilon_{\text {yield }} \ll 1$ relative to those retained.

The crust is effectively a perfect electrical conductor at seismic frequencies, so the perturbed electric field is given by

$$
\delta \boldsymbol{E}=-\frac{1}{c} \frac{\partial \xi}{\partial t} \times \boldsymbol{B} .
$$

Equation (12), combined with Maxwell's equations,

$$
\nabla \times \delta \boldsymbol{E}=-\frac{1}{c} \frac{\partial \delta \boldsymbol{B}}{\partial t}
$$

and

$$
\nabla \times \delta B=\frac{4 \pi}{c} \delta \boldsymbol{j}+\frac{1}{c} \frac{\partial \delta \boldsymbol{E}}{\partial t},
$$

may be used to relate $\delta \boldsymbol{j}$ to $\xi$.

The transmission coefficient for the energy flux between the crust and the magnetosphere should not be significantly affected by the polarization and propagation angle of the incident shear wave. We consider then the simplest possible case, a vertically propagating shear wave polarized in such a way that $\xi \propto z \times B$. For this special case $\nabla \cdot \xi, \delta \rho$, and $\delta p$ all vanish. Next, we take a harmonic time dependence, $\exp (-i \omega t)$, for the perturbation variables and combine equations (9)-(14) to derive the wave equation,

$$
\frac{d}{d z}\left(\tilde{\mu} \frac{d \xi}{d z}\right)+\tilde{\rho} \omega^{2} \xi=0,
$$

where $\tilde{\mu}$ and $\tilde{\rho}$ are the effective shear modulus and density:

$$
\tilde{\mu} \equiv \mu+\frac{(B \cos \alpha)^{2}}{4 \pi},
$$

and

$$
\tilde{\rho} \equiv \rho+\frac{B^{2}}{4 \pi c^{2}} .
$$

Here $\cos \alpha \equiv B_{z} / B$. Equations (2) and (3) are used to obtain the dependence of $\mu$ and $\rho$ on depth. The magnetoelastic wave speed, $\tilde{v}_{s}$, is equal to $(\tilde{\mu} / \tilde{\rho})^{1 / 2}$.

Deep in the crust, but above neutron drip, equation (15) has the asymptotic solution ${ }^{3}$

$$
\xi \sim|z|^{-7 / 4}\left\{\exp [-i(u+\omega t)]+A_{R} \exp [i(u-\omega t)]\right\},
$$

\footnotetext{
${ }^{3}$ The $z$-dependence of the amplitude follows from conservation of the WKBJ flux, $F \sim \rho v_{s}(\omega \xi)^{2}$.
}

where

$$
u \equiv-\int^{z} d z \frac{\omega}{v_{s}}=\frac{6.5}{Z^{1 / 3}}\left(\frac{\hbar c}{e^{2}} \frac{\omega^{2}|z|}{g}\right)^{1 / 2} .
$$

The first and second terms on the right-hand side of equation (18) represent the upward-propagating, incident wave and the downward-propagating, reflected wave, with $A_{R}$ the complex reflection amplitude.

In the magnetosphere, $z>0, \tilde{\mu}=(B \cos \alpha)^{2} /(4 \pi), \tilde{\rho}=B^{2} /$ $\left(4 \pi c^{2}\right)$, and the wave equation reduces to that for relativistic Alfvén waves,

$$
\frac{d^{2} \xi}{d z^{2}}+\sec ^{2} \alpha \frac{\omega^{2}}{c^{2}} \xi=0 .
$$

The outward-propagating solution has the form

$$
\xi=A_{T} \exp \left[i \omega\left(\sec \alpha \frac{z}{c}-t\right)\right],
$$

where $A_{T}$ is the complex transmission amplitude.

To determine $A_{R}$ and $A_{T}$, we perform two numerical integrations of equation (15) from $z \ll 0$ to $z>0$ starting from different lower boundary conditions. Then we choose the appropriate linear combination of the two boundary conditions in such a way that the solution satisfies the radiation condition, $\xi^{\prime}=i \omega(\sec \alpha) \xi / c$, for $z>0$. The transmission coefficient, $T$, the ratio of the transmitted to the incident energy flux, is given by

$$
T=1-\left|A_{R}\right|^{2} .
$$

The solid curve in Figure 1 displays $T$ as a function of frequency for various vertical magnetic field strengths. These numerical results may be understood with reasonable quantitative accuracy as follows.

For a homogeneous crust, it is easy to show that the transmission coefficient for a vertically propagating shear wave is given by the familiar formula

$$
T=\frac{4 Z_{C} Z_{M}}{\left(Z_{C}+Z_{M}\right)^{2}},
$$

where $Z_{C}$ and $Z_{M}$ are the impedances of the crust and the magnetosphere. Each impedance is the product of the relevant propagation speed and effective density. Thus,

$$
Z_{C}=(\tilde{\rho} \tilde{\mu})^{1 / 2} \quad \text { and } \quad Z_{M}=\frac{B^{2} \cos \alpha}{4 \pi c} .
$$

To apply equation (23) to an inhomogeneous crust, we need to know where to evaluate $Z_{c}$. It turns out that there are two different regimes to consider. To proceed, we define a new dependent variable $\Psi$ by

$$
\Psi \equiv \tilde{\mu}^{1 / 2} \xi
$$

Then the wave equation (15) becomes

$$
\frac{d^{2} \Psi}{d z^{2}}+\left[\frac{1}{4 \tilde{\mu}^{2}}\left(\frac{d \tilde{\mu}}{d z}\right)^{2}-\frac{1}{2 \tilde{\mu}} \frac{d^{2} \tilde{\mu}}{d z^{2}}+\frac{\omega^{2} \tilde{\rho}}{\tilde{\mu}}\right] \Psi=0 .
$$

The expression in square brackets is positive at large depth in the crust and also in the magnetosphere, so in these regions the 


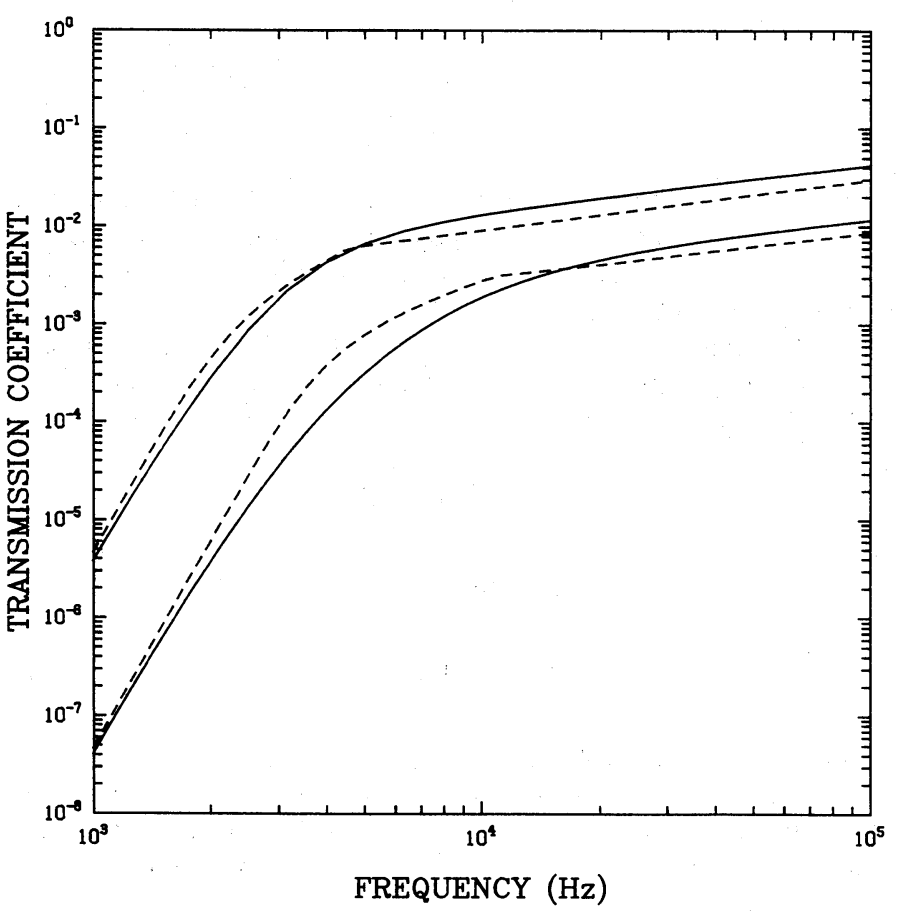

FIG. 1.-Transmission coefficient as a function of frequency for a vertically propagating wave. The solid lines refer to numerical calculations, while the dashed lines show the quasi-analytical approximations discussed in the text. The upper and lower pairs of curves are for $10^{12}$ and $10^{11} \mathrm{G}$ fields, respectively.

waves are propagating in a WKBJ sense. In between there is an evanescent zone in which $\xi$ is nearly constant and the strain is very small. This illustrated in Figure 2, which displays the displacement amplitude and strain as calculated numerically for $v=10^{4} \mathrm{~Hz}$ and $B=10^{11} \mathrm{G}$.

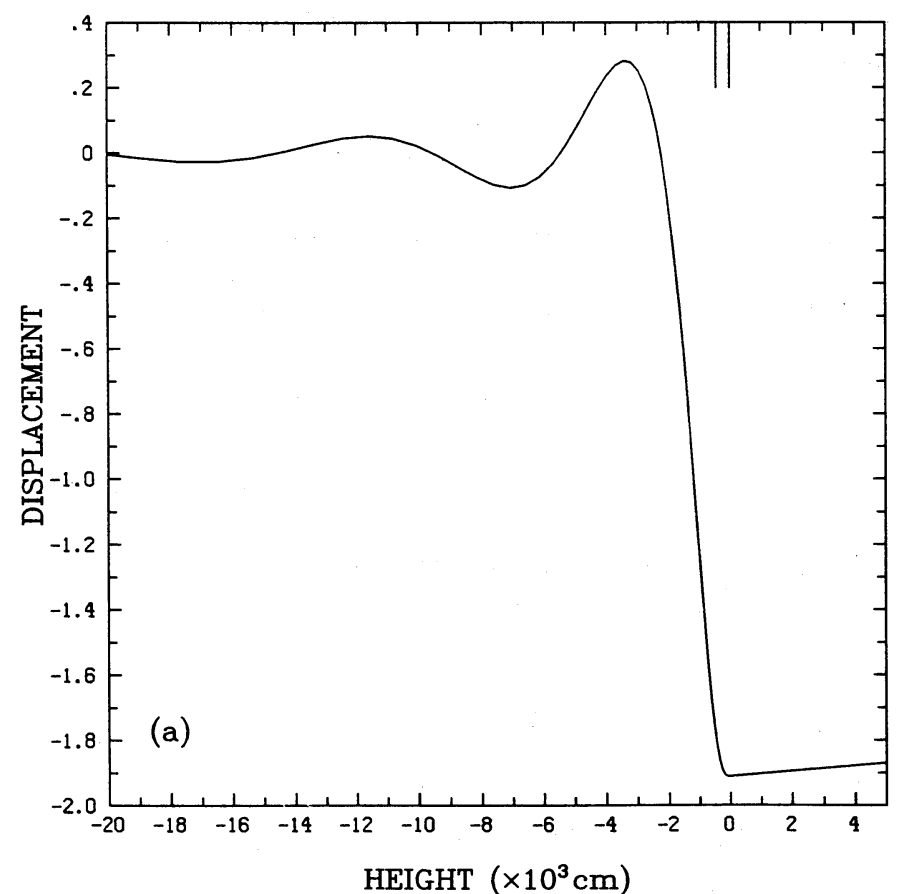

For $v \ll 10^{4} \mathrm{~Hz}$, equation (23) yields a reasonably accurate formula for $T$ if $Z_{C}$ is evaluated at $z_{\text {ev }}$, the lower boundary of the evanescent zone, where the expression in square brackets in equation (26) vanishes. At low frequencies, $z_{\mathrm{ev}}$ is in the region where the electrons are relativistic. In this regime

$$
\left|z_{\text {ev }}\right| \simeq 4 \times 10^{4}\left(\frac{v}{10^{3} \mathrm{~Hz}}\right)^{-2} \mathrm{~cm},
$$

and equations (23) and (24) imply

$$
T \simeq \frac{4 Z_{M}}{Z_{C}} \simeq 5 \times 10^{-8}\left(\frac{B}{10^{11} \mathrm{G}}\right)^{2}\left(\frac{v}{10^{3} \mathrm{~Hz}}\right)^{7} \cos \alpha .
$$

At high frequencies, substantial reflection occurs below $z_{\mathrm{ev}}$, where the propagation speed increases rapidly with height as the magnetic field starts to dominate the shear stress and the density decreases. In this case, it is more appropriate to evaluate $Z_{C}$ where the vertical wavenumber is changing on the scale of a wavelength, or, equivalently, where

$$
\frac{d \tilde{v}_{s}^{2}}{d z}= \pm \omega \tilde{v}_{s}
$$

For $v \gg 10^{4} \mathrm{~Hz}$, this leads to the following approximation for the transmission coefficient:

$$
T \simeq 9 \times 10^{-3}\left(\frac{B}{10^{11} \mathrm{G}}\right)^{4 / 7}\left(\frac{v}{10^{5} \mathrm{~Hz}}\right)^{3 / 7}(\cos \alpha)^{-3 / 7} .
$$

The dashed lines in Figure 1 show the approximate solution for the transmission coefficient as calculated from equation (23) with $Z_{C}$ evaluated using the relevant criterion as described above. The approximate formulae (28) and (30) for $T$ are quite accurate in both the low- and the high-frequency limits.

We have seen that only a small fraction $T$ of the incident

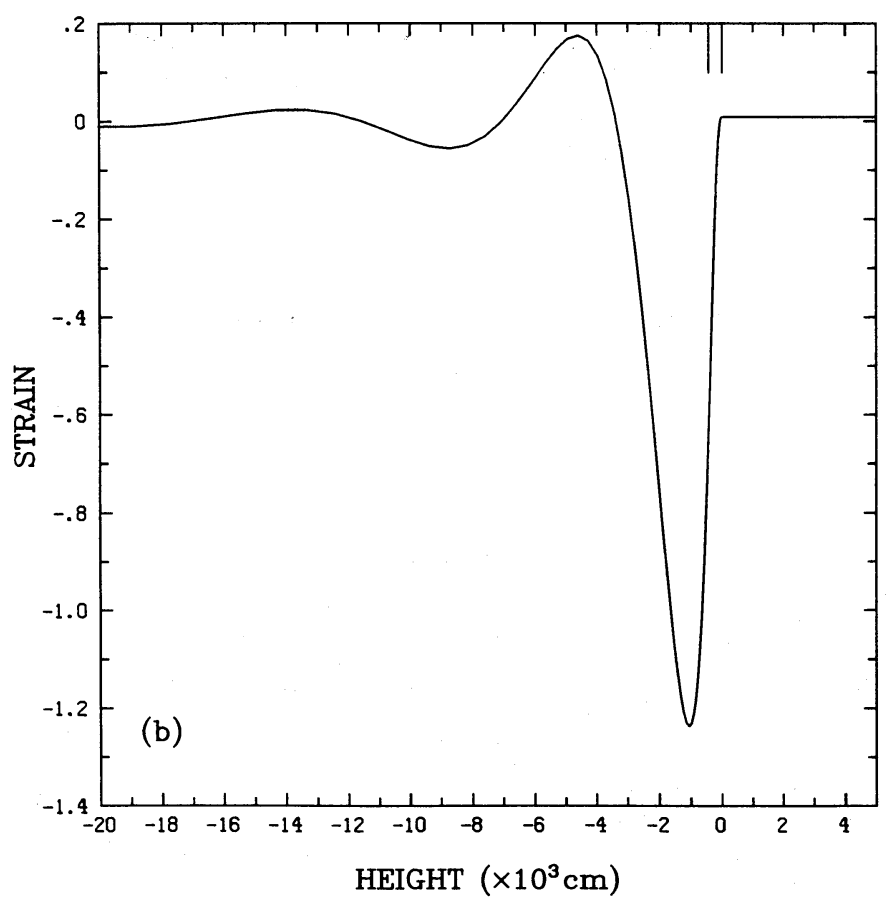

FIG. 2. - (a) Displacement amplitude and (b) associated strain, in arbitrary units as a function of height above the surface for a $10^{4} \mathrm{~Hz}$ vertically propagating wave in a $10^{11} \mathrm{G}$ magnetic field. The location of the evanescent zone is indicated by the pair of vertical lines. 
wave flux leaks into the magnetosphere. The fate of the reflected energy is clearly of interest. The angular momentum barrier ${ }^{4}$ and an increase in wave speed with depth both refract waves upward. This behavior is an obvious consequence of the WKBJ dispersion relation,

$$
k_{z}^{2}(z)=\left(\frac{\omega}{v_{s}}\right)^{2}-\frac{l(l+1)}{r^{2}} .
$$

Compressional waves are guaranteed to return eventually to the surface, since they propagate throughout the star. However, shear waves cannot propagate in the fluid interior below the crust. Those which reach this region may be reflected if the rigidity drops sufficiently abruptly to zero at the inner boundary of the crust. Otherwise, as the waves slow down, their radial wavevectors and amplitudes will increase, and their energy will ultimately be dissipated as heat.

The waves which do return will bounce many times off the surface and will spread throughout the crust on a time scale $\sim 10^{-2}\left[v_{s}\left(z_{Q}\right) /\left(2 \times 10^{8} \mathrm{~cm} \mathrm{~s}^{-1}\right)\right]^{-1} \mathrm{~s}$. The characteristic storage time for wave energy in the crust, $\tau(v)$, is twice the time it takes the waves to cross the crust, $2 t_{c}$, divided by $T(v)$. For vertically propagating waves,

$$
\tau(v) \sim-\frac{2}{T(v)} \int_{0}^{z_{Q}} \frac{d z}{v_{s}} \sim \frac{5 \times 10^{-6}\left|z_{Q}\right|^{1 / 2}}{T(v)} \mathrm{s} .
$$

At low frequencies, equations (28) and (32) imply

$$
\tau \sim \frac{3 \times 10^{4}}{\cos \alpha}\left(\frac{v}{10^{3} \mathrm{~Hz}}\right)^{-7}\left(\frac{B}{10^{11} \mathrm{G}}\right)^{-2}\left(\frac{\left|z_{Q}\right|}{10^{5} \mathrm{~cm}}\right)^{1 / 2} \mathrm{~s} .
$$

while at high frequencies equations (30) and (32) give

$$
\tau \sim 0.2(\cos \alpha)^{3 / 7}\left(\frac{v}{10^{5} \mathrm{~Hz}}\right)^{-3 / 7}\left(\frac{B}{10^{11} \mathrm{G}}\right)^{-4 / 7}\left(\frac{\left|z_{Q}\right|}{10^{5} \mathrm{~cm}}\right)^{1 / 2} \mathrm{~s} .
$$

With the same scalings, $\tau \sim 1 \mathrm{~s}$ at $v_{0}=10^{4} \mathrm{~Hz}$.

To obtain a crude picture of the time-dependent Alfvén wave luminosity following a quake, we combine the seismic wave power spectrum given by equation (8) with $\tau(v)$ from equation (32). A trivial calculation yields

$$
L\left(t>t_{c}\right)=\int_{0}^{\infty} d v \frac{E_{v}}{\tau(v)} \exp \left[-\frac{t-t_{c}}{\tau(v)}\right] .
$$

A graph of $L(t)$ for a nominal quake with a total energy release of $10^{38}$ ergs is presented in Figure 3.

Our analysis of the propagation of shear waves has neglected damping. In the absence of empirical data, it is difficult to identify the principal damping mechanisms, let alone to estimate the seismic $Q$ 's they provide. Since the storage time for the seismic energy in the crust is of order $10^{s}$ wave periods, any process which produces $Q \lesssim 10^{4}$ would be important.

For the Earth, the seismic $Q$ ranges from $\sim 100$ in the upper mantle to $\sim 200$ in the crust to $\sim 500$ in the deep mantle. The damping mechanism is not well established, but stress-induced motion of dislocations is a plausible guess (Minster 1980). This mechanism is temperature-dependent, and gives rise to $Q$ 's which decrease as the melting temperature is approached.

The stress-induced motion of dislocations may well domi-

\footnotetext{
${ }^{4} \mathrm{Or}$, equivalently, the $1 / r$ dependence of the horizontal wavenumber in a spherical star.
}

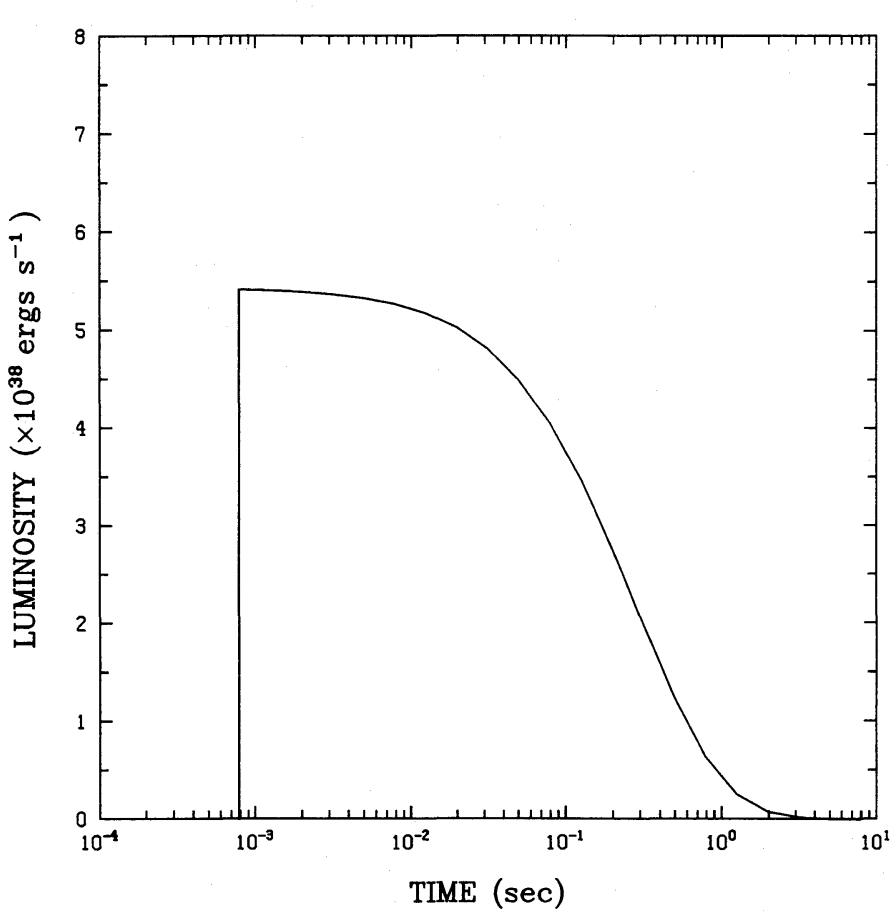

Fig. 3.-Alfvén wave luminosity as a function of time, normalized to a total seismic energy release of $10^{38}$ ergs. The energy starts to be transmitted into the magnetosphere after a time $t_{c} \sim 8 \times 10^{-4} \mathrm{~s}$.

nate the damping of shear waves in the crusts of neutron stars. However, it is not possible to make a reliable estimate of the $Q$ 's it produces.

Theoretical calculations of the degenerate electron viscosity in a solid neutron star crust have been reported by Flowers and Itoh (1976). They included electron scattering by phonons, impurity ions, other electrons, and free neutrons. We neglect chemical impurities because of the large uncertainty in their concentration and type. For core temperatures of $\sim 10^{6} \mathrm{~K}$, the damping due to electron viscosity is most severe at high densities, the dominant scattering mechanism being due to phonons. At neutron drip, the damping time is of order $4 \times 10^{3} \mathrm{~s}$ for $10^{4} \mathrm{~Hz}$ waves. This is probably an underestimate because of the likely presence of other sources of electron scattering, especially lattice imperfections. Even so, it is still much longer than the typical time scale for a $\gamma$-ray burst.

Itoh, Kohyama, and Takeuchi (1987) have calculated the ionic contribution to the viscosity for the liquid state. Their results give a damping time of order $300 \mathrm{yr}$ for densities of $10^{6}$ $\mathrm{g} \mathrm{cm}^{-3}$. Although those mechanisms which can be quantitatively analyzed provide negligible damping on $\gamma$-ray burst time scales, it should be borne in mind that this does not constitute a proof that damping is unimportant.

Up to this point we have considered how a vertically propagating shear wave polarized orthogonal to the ambient magnetic field couples to an Alfvén wave. Since the magnetosphere can also support fast magnetosonic waves, it is natural to inquire whether more general seismic disturbances couple to them. As a preliminary step to addressing this query, we note that the dispersion relation for fast magnetosonic waves is isotropic and reads

$$
\omega^{2}=(c k)^{2}
$$

which differs from the anisotropic dispersion relation for 
Alfvén waves,

$$
\omega^{2}=(c k \cos \psi)^{2} \text {. }
$$

Here, $k$ is the magnitude of the wavevector, and $\psi$ is the angle between $\boldsymbol{k}$ and $\boldsymbol{B}$. (This dispersion relation, derived assuming an infinite conductivity, is also valid for a low-density, relativistic plasma, as shown in Appendix A.) The constancy of $\omega$ and the horizontal component of $k, k \sin \theta$, across the surface leads to Snell's law. For fast magnetosonic waves and Alfvén waves, the appropriate forms are

$$
\frac{\sin \theta_{t}}{\sin \theta_{i}}=\frac{c}{\tilde{v}_{s}}
$$

and

$$
\frac{\sin \theta_{t}}{\sin \theta_{i}}=\frac{c \cos \psi}{\tilde{v}_{s}}
$$

Here, $\theta_{i}$ and $\theta_{t}$ denote the angles of incidence and transmission. At the reflecting layer, $\tilde{v}_{s} \ll c$, and thus only waves having very small angles of incidence can couple to fast magnetosonic waves. For Alfvén waves the only restriction is that the transmitted wavevector be almost orthogonal to $\boldsymbol{B} .^{5}$

The displacement amplitude of a propagating shear wave increases with height in the crust as a consequence of the conservation of energy flux, ${ }^{6}$ before saturating at a constant value in the evanescent zone. Because the strain amplitude, $\epsilon$, is proportional to the gradient of the displacement, and the magnitude of the wave vector, $k=\omega / \tilde{v}_{s}$, also increases with height, $\epsilon$ reaches a maximum near the bottom of the evanescent zone (cf. Fig. 2b). The following argument relates the maximum strain amplitude to the Alfvén wave luminosity.

We assume that the entire neutron star surface radiates Alfvén waves. Then the fractional perturbation of the surface magnetic field associated with luminosity $L$ is

$$
\frac{\delta B}{B} \sim 6 \times 10^{-4}\left(\frac{L}{10^{38} \mathrm{ergs} \mathrm{s}^{-1}}\right)^{1 / 2}\left(\frac{B}{10^{11} \mathrm{G}}\right)^{-1} .
$$

In terms of the surface displacement amplitude, equations (12) and (13) imply

$$
\frac{\delta B}{B} \sim \frac{\omega}{c} \xi
$$

Thus, we estimate the total strain at $z_{\mathrm{ev}}$ to be

$$
\epsilon \sim 0.5\left[\frac{v_{s}\left(z_{\mathrm{ev}}\right)}{4 \times 10^{7} \mathrm{~cm} \mathrm{~s}^{-1}}\right]^{-1}\left(\frac{L}{10^{38} \mathrm{ergs} \mathrm{s}^{-1}}\right)^{1 / 2}\left(\frac{B}{10^{11} \mathrm{G}}\right)^{-1} \text {. }
$$

With the chosen scaling, the maximum strain is dangerously close to unity. More accurate numerical calculations for vertically incident $10^{4} \mathrm{~Hz}$ waves, with the same scaling parameters as in equation (42), predict that the maximum strain amplitude is 0.2 , and that it occurs at $z \approx 2 z_{\mathrm{ev}}$. A dynamic yield strain as large as 0.1 is not unusual (R. Jeanloz 1988, private

\footnotetext{
${ }^{5}$ We discuss coupling to fast magnetosonic waves and its relation to previous calculations of electromagnetic damping in Appendix B.

${ }^{6}$ The flux, $F$, is proportional to $\tilde{\rho} \tilde{v}_{s} \xi^{2}$, and $\tilde{\rho} \tilde{v}_{s}$ decreases with height.
}

communication). Thus, we cannot predict whether shear waves transfer their energy to Alfvén waves and/or crumble the crust, generating heat. However, it is clear that the neutron starquake model is not viable if bursts are significantly more distant, and consequently more luminous, than estimated above.

Suppose that some heat is deposited at $|z|_{\mathrm{ev}} \sim 450 \mathrm{~cm}$ (this is the appropriate value of $z_{\mathrm{ev}}$ for $v \sim 10^{4} \mathrm{~Hz}$ ), where $\rho \sim 9 \times 10^{5} \mathrm{~g} \mathrm{~cm}^{-3}$. At this depth, the Debye temperature is $\sim 3 \times 10^{6} \mathrm{~K}$, and the melting temperature is $\sim 3 \times 10^{7} \mathrm{~K}$. A heat input of $\gtrsim 10^{35}$ ergs will melt the solid crust.

The transport of heat from $z_{\mathrm{ev}}$ to the nondegenerate outer layers is controlled by degenerate-electron conduction. The diffusion time scale through a liquid crust is given by

$$
t_{\mathrm{diff}} \sim \frac{z_{\mathrm{ev}}^{2} \rho C_{v}}{K Z \mu_{e} m_{u}} \sim 10^{3} \mathrm{~s},
$$

where $C_{v}$ is the capacity per ion and $K$ is the thermal conductivity, the dominant electron scattering mechanism being due to ions (Flowers and Itoh 1976). Thus thermal energy will be radiated from the surface as $\mathrm{X}$-rays, on time scales which are much longer than the typical duration of the $\gamma$-ray emission, as required by the X-ray paucity constraint.

\section{ALFVÉN WAVES IN THE MAGNETOSPHERE}

An attempt to predict the behavior of Alfven waves in the magnetosphere is fraught with uncertainties. Below we touch on a few of the most critical issues.

The dispersion relation given by equation (37) implies that the group velocity of Alfvén waves has magnitude $c$ and is aligned with the local magnetic field. Close to the stellar surface, the Alfvén waves excited by a quake have propagation vectors that are nearly orthogonal to the magnetic field. As the waves travel out along the curved field lines, the wavevectors rotate toward alignment or counteralignment with the magnetic field lines, with the component of $\boldsymbol{k}$ along $\boldsymbol{B}$ fixed by the constancy of $\omega$. Significant rotation of $\boldsymbol{k}$ occurs over distances comparable to the radii of curvature of the field lines. This result may be derived in a straightforward fashion from the eikonal equations (Weinberg 1962). Physically, it arises because the Alfvén wave phase velocity is proportional to $|\cos \psi|$, where $\psi$ is the angle between $\boldsymbol{k}$ and $\boldsymbol{B}$. Consequently, an initially planar wave front which travels out along a bundle of diverging field lines bows out because the central part of the wave front propagates faster than the outer parts.

We have already discussed the magnetic field perturbations required for Alfvén waves to carry the luminosity of a typical $\gamma$-ray burst. The energy emitted by the star is guided along magnetospheric field lines and might bounce many times between their feet, at least for closed field lines. Since the energy flux transmission coefficient between crust and magnetosphere is the same in both directions, the equilibrium energy density of Alfvén waves near the stellar surface, $\sim 10^{17}\left(E_{Q} / 10^{38}\right.$ ergs) ergs $\mathrm{cm}^{-3}$, is smaller by $\tilde{v}_{s} / c \lesssim 10^{-3}$ than the energy density of seismic waves just below the evanescent zone in the crust. The equilibrium Alfvén wave energy density corresponds to a perturbed magnetic field strength

$$
\frac{\delta B}{B} \sim 10^{-2}\left(\frac{E_{Q}}{10^{38} \mathrm{ergs}}\right)^{1 / 2}\left(\frac{B}{10^{11} \mathrm{G}}\right)^{-1} \mathrm{G}
$$

near the star. This value of $\delta B$ is significantly larger than that obtained from the Alfven wave luminosity in equation (40). 
The larger value of $\delta B$ given in equation (44) arises from waves propagating in both directions along the field lines.

Because Alfvén waves transport energy without loss along the equilibrium field lines, their relative amplitudes vary according to

$$
\frac{\delta B}{B} \propto B^{-1 / 2}
$$

Equations (44) and (45) indicate that, if equilibrium is reached, the Alfvén waves are likely to become nonlinear not very far from the star.

Up to this point, we have been making the implicit assumption that the plasma density in the magnetosphere is high enough so that the MHD limit applies to Alfvén wave propagation. We shall now show that this is a questionable assumption.

The corotation charge number density (Goldreich and Julian 1969),

$$
n_{\mathrm{cr}} \simeq \frac{B}{P c e} \simeq 7 \times 10^{9}\left(\frac{B}{10^{11} \mathrm{G}}\right)\left(\frac{P}{1 \mathrm{~s}}\right)^{-1} \mathrm{~cm}^{-3},
$$

sets a lower limit on the magnetospheric plasma density, $n$. Here, $P$ is the rotational period of the neutron star. For planetary magnetospheres, $n \gg n_{\mathrm{cr}}$; neutral plasma is injected into the magnetosphere from the upper atmosphere of the planet, from satellites, and from the solar wind. For quiescent old neutron stars, it is plausible that $n \approx n_{\mathrm{cr}}$, since the atmospheric scale height is very small, $\sim 0.1 \mathrm{~cm}$ for a surface temperature $T_{s}=10^{5} \mathrm{~K}$. Much higher densities may exist if accretion is occurring, or if an energetic process expels plasma from the stellar atmosphere. In the latter context, it is noteworthy that substantial plasma may be torn off the surface by outwardpropagating Alfvén waves. In the presence of Alfvén waves, the force on charged particles is $\sim e \delta B \sim 5 \times 10^{-10}(\delta B / G)$ dynes, which is much larger than the gravitational force on a proton, $10^{-10}$ dynes, at the surface.

For low plasma densities a different nonlinearity from that discussed previously may occur even at relative field amplitudes $\delta B / B \ll 1$. Because the wavevector is nearly orthogonal to the magnetic field near the surface, equation (12) implies that the wavevector is nearly parallel to $\delta E$. Therefore, the displacement current and $\boldsymbol{\nabla} \times \delta \boldsymbol{B}$ are nearly orthogonal. From equation (14), we see that a substantial physical current is necessary to support the wave. If the plasma density is so low that the required drift velocities exceed $c$, the wave is charge-starved. This occurs where

$$
\frac{\delta B}{B} \sim \frac{2 n e c}{v_{0} B} \sim 10^{-4}\left(\frac{n}{n_{\mathrm{cr}}}\right)\left(\frac{P}{1 \mathrm{~s}}\right)^{-1} .
$$

Based on the values of $\delta B / B$ given by equations (40) and (44), the Alfvén waves are expected to be charge-starved close to the stellar surface unless $n \gg n_{\mathrm{cr}}$. The fate of such waves requires further investigation.

Once the Alfvén waves go nonlinear, by whatever mechanism, a substantial part of the wave energy is probably transferred to the electrons. An upper limit to the Lorentz factors is obtained by balancing the electrostatic acceleration with curvature radiation reaction. We find

$$
\gamma \sim 10^{7}\left(\frac{\delta B / B}{10^{-4}}\right)^{1 / 4}\left(\frac{B}{10^{11} \mathrm{G}}\right)^{1 / 4}\left(\frac{R}{10^{6} \mathrm{~cm}}\right)^{1 / 2} .
$$

\section{SOURCES OF FREE ENERGY}

We deduce a mean repetition time between $\gamma$-ray bursts of

$$
\tau \sim 10^{4} f\left(\frac{n}{3 \times 10^{-4} \mathrm{pc}^{-3}}\right)\left(\frac{D}{1 \mathrm{kpc}}\right)^{3}\left(\frac{\dot{N}}{100 \mathrm{yr}^{-1}}\right)^{-1} \mathrm{yr}
$$

for each neutron star under the assumption that all neutron stars produce $\gamma$-ray bursts during a fraction $f$ of their lifetimes. Here, $n$ is the local density of neutron stars and $\dot{N}$ is the number of bursts observed per year. The scaling for $n$ comes from Lyne, Manchester, and Taylor (1985), who find that the Galactic pulsar birthrate could be as high as $3 \times 10^{-11} \mathrm{pc}^{-2}$ $\mathrm{yr}^{-1}$. We adopt a lifetime of $10^{10} \mathrm{yr}$ and a vertical scale height of $1 \mathrm{kpc}$ for old neutron stars.

The total amount of energy released by each star is

$$
\begin{aligned}
E_{\mathrm{tot}} \sim \frac{10^{44}}{\eta}\left(\frac{\dot{N}}{100 \mathrm{yr}^{-1}}\right)\left(\frac{F}{10^{-6} \mathrm{ergs} \mathrm{cm}^{-2}}\right) \\
\quad \times\left(\frac{n}{3 \times 10^{-4} \mathrm{pc}^{-3}}\right)^{-1}\left(\frac{D}{1 \mathrm{kpc}}\right)^{-1} \text { ergs } .
\end{aligned}
$$

It seems inescapable that, associated with quake activity, an energy at least comparable to $E_{\text {tot }}$ must be dissipated as heat in the neutron star interior. If most of this energy is ultimately radiated from the stellar surface, it would account for a surface temperature $T_{\text {quake }} \sim 2 \times 10^{4} \mathrm{~K}$.

The source of free energy to power quakes in neutron stars is a mystery. During its birth a neutron star radiates away $\sim 10^{53}$ ergs of binding energy. Thus, it seems possible that old neutron stars might retain as much as $10^{44}$ ergs of free energy. However, upon closer examination there are not many places where this amount of free energy could be stored, and even fewer from which its release would produce quakes. We have already seen in $\S$ II that the crust can store at most $\sim 10^{44}$ ergs of elastic energy. Other sources are considered below.

The rotational energy is $E_{R} \sim 10^{46} P^{-2}$ ergs, where $P$ is the period in seconds. If the star rotates rigidly as it is spun down, only a tiny fraction, $\lesssim 10^{-11}$, of this energy would be stored elastically. Internal differential rotation is a source of free energy whose sudden release has been proposed as an explanation for pulsar glitches (Pines and Alpar 1985). Consider a star with two components of fixed moments of inertia $I_{1}$ and $I_{2}$ which are rotating with angular velocities $\Omega$ and $\Omega+\Delta \Omega$, respectively. The amount of energy which can be released by bringing the star into uniform rotation while conserving total angular momentum is

$$
\Delta E_{R}=\frac{I_{1} I_{2}}{2\left(I_{1}+I_{2}\right)} \Delta \Omega^{2} .
$$

The fact that radio pulsars are such excellent clocks is most easily understood if they have small internal differential rotation. In fact, it is generally believed that the anisotropic neutron superfluid in the core rotates rigidly with the crust as a result of strong electromagnetic coupling. The pinning of vortex lines in the crust allows the isotropic crustal neutron superfluid to rotate more rapidly than the crustal lattice. Unpinning events are associated with glitches. In this picture $I_{1}$ and $I_{2}$ correspond to the crustal lattice plus core superfluid and to the crust superfluid, respectively. Typically $I_{2} / I_{1} \sim$ $10^{-2}$. Theoretical estimates of the maximum value of $\Delta \Omega$ which can be supported by the pinning force range from 0.1 to 10 radians $\mathrm{s}^{-1}$ (Alpar et al. 1984). Individual burst events 
might therefore be explained in this way. The total amount of free energy available over the lifetime of the bursting neutron star is equal to the initial rotational energy of the crustal superfluid. An initial angular velocity $\gtrsim 10$ radians $s^{-1}$ would be sufficient to explain the total burst energetics.

An alternative is that differential rotation might temporarily store energy by winding up an internal magnetic field. The rate of change of magnetic field energy is

$$
\frac{d E_{B}}{d t}=T_{\text {ext }} \Omega-I_{1} \Omega \dot{\Omega}-I_{2}(\Omega+\Delta \Omega)(\dot{\Omega}+\Delta \dot{\Omega}),
$$

where $T_{\text {ext }}$, the external torque applied to the crust, gives the total rate of change of angular momentum of the star,

$$
T_{\text {ext }}=I_{1} \dot{\Omega}+I_{2}(\dot{\Omega}+\Delta \dot{\Omega}) \text {. }
$$

Equations (52) and (53) imply that the ratio of the change of magnetic field energy to that of the rotational energy is

$$
\frac{d E_{B}}{d E_{R}}=-\frac{I_{2}}{I_{1}+I_{2}} \frac{\Delta \Omega}{\Omega},
$$

for $|\Delta \Omega / \Omega| \ll 1$ and $\mid \Delta \Omega / \Omega) \ll 1$, showing that this is an unlikely source of free energy.

Phase transitions which occur at temperature $T$ could release energy $\sim N k T$, where $N$ is the total number of particles involved. However, old neutron stars are thought to be cold, $T \lesssim 10^{6} \mathrm{~K}$, so over their lifetimes at least $0.1 \%$ of their mass would have to be involved in phase transitions for them to release $\sim 10^{44}$ ergs.

Little is understood concerning the strength and structure of internal magnetic fields in neutron stars. Because of the high electrical conductivity of degenerate material, large fields might persist for the lifetimes of the stars. Since magnetic fields are buoyant, they could produce an anisotropic stress on the crust (Sturrock 1986). Interior fields of order $10^{14} \mathrm{G}$ would be sufficient to explain the total $\gamma$-ray burst energetics.

Having seen how limited the sources of free energy in neutron star interiors are, we consider the obvious external source, the accretion of interstellar gas. Hydrodynamic accretion from the interstellar medium onto a moving neutron star proceeds at the rate (Alcock and Illarionov 1980)

$$
\dot{M} \sim 3 \times 10^{9}\left(\frac{M}{M_{\odot}}\right)\left(\frac{v}{50 \mathrm{~km} \mathrm{~s}^{-1}}\right)^{-3}\left(\frac{n_{\infty}}{1 \mathrm{~cm}^{-3}}\right) \mathrm{g} \mathrm{s}^{-1},
$$

where $M$ is the star's mass, $v$ is the speed at which it is moving, and $n_{\infty}$ is the hydrogen number density of the interstellar gas. The resulting stellar surface temperature is

$$
\begin{aligned}
T_{\text {acc }} \sim 1.5 \times 10^{5}\left(\frac{M}{M_{\odot}}\right)^{1 / 4}\left(\frac{\dot{M}}{3 \times 10^{9} \mathrm{~g} \mathrm{~s}^{-1}}\right)^{1 / 4} & \\
& \times\left(\frac{R_{s}}{10 \mathrm{~km}}\right)^{-3 / 4} \mathrm{~K},
\end{aligned}
$$

where $R_{s}$ is the neutron star radius. The incoming material does not fall directly onto the star's surface unless the Alfvén radius,

$$
\begin{aligned}
r_{\mathrm{A}} \sim 10^{10}\left(\frac{B}{10^{11} \mathrm{G}}\right)^{4 / 7} & \left(\frac{\dot{M}}{3 \times 10^{9} \mathrm{~g} \mathrm{~s}^{-1}}\right)^{-2 / 7} \\
& \times\left(\frac{M}{M_{\odot}}\right)^{-1 / 7}\left(\frac{R_{s}}{10 \mathrm{~km}}\right)^{12 / 7} \mathrm{~cm},
\end{aligned}
$$

is smaller than the corotation radius, $r_{\mathrm{cr}}$, at which the orbital period is equal to the star's spin period:

$$
r_{\mathrm{cr}} \sim 10^{8}\left(\frac{P}{1 \mathrm{~s}}\right)^{2 / 3} \mathrm{~cm} .
$$

With our scalings, $r_{\mathrm{cr}}>r_{\mathrm{A}}$ for $P \gtrsim 500 \mathrm{~s}$. If $r_{\mathrm{cr}}<r_{\mathrm{A}}$, unaccreted material gains angular momentum causing the rotational energy of the neutron star to decay at the rate

$$
\dot{E}_{P} \sim 10^{28}\left(\frac{\dot{M}}{3 \times 10^{9} \mathrm{~g} \mathrm{~s}^{-1}}\right)\left(\frac{r_{\mathrm{A}}}{10^{10} \mathrm{~cm}}\right)^{1 / 2}\left(\frac{P}{1 \mathrm{~s}}\right)^{-1} \operatorname{ergs~s}^{-1} .
$$

This is known as the propeller mechanism (Illarionov and Sunyaev 1975).

Energy is also lost through magnetic braking at a rate

$$
\dot{E}_{M} \sim 6 \times 10^{29}\left(\frac{B}{10^{11} \mathrm{G}}\right)^{2}\left(\frac{P}{1 \mathrm{~s}}\right)^{-4} \operatorname{ergs~s}^{-1} .
$$

The propeller mechanism dominates magnetic braking for $P \gtrsim 4 \mathrm{~s}$, and the neutron star spins down enough to permit accretion on a time scale $\sim 10^{8} \mathrm{yr}$.

The mass accreted over the burst repetition time scale $\tau$ is $M_{a} \sim 10^{21} \mathrm{~g}$, and covers an area $A \sim 3 \times 10^{8} \mathrm{~cm}^{2}$, provided that the magnetic field confines the accretion flow above and below the star surface. The strain built up in the crust is given by

$$
\begin{aligned}
\epsilon_{a} \sim 10^{-2}\left[\frac{\mu\left(z_{Q}\right)}{10^{28} \mathrm{ergs} \mathrm{cm}^{-3}}\right]^{-1}\left(\frac{A}{3 \times 10^{8} \mathrm{~cm}^{2}}\right)^{-1} \\
\times\left(\frac{\dot{M}}{3 \times 10^{9} \mathrm{~g} \mathrm{~s}^{-1}}\right)\left(\frac{\tau}{10^{4} \mathrm{yr}}\right),
\end{aligned}
$$

which might be large enough to trigger crustquakes. However, it is by no means clear that accretion, even if anisotropic, will stress the crust to the level calculated in equation (61). The upper crust is not very strong, and mass loading by accretion seems likely to force a sideways flow of material which would limit the stress built up at depth.

Accretion also leads to the storage of free energy in nuclear form. The weight of the accreted material causes the equilibrium phase boundaries separating different nuclear compositions to rise relative to the actual phase boundaries. The metastable nuclei so produced may undergo occasional, sudden transitions to their equilibrium states. The mass between the equilibrium and actual phase boundary must equal that accreted since the previous adjustment at that phase transition. Because around $1 \mathrm{MeV}$ per nucleon can be released during a phase change, seismic events with $E \lesssim 10^{39}$ ergs might be produced on the burst repetition time scale. ${ }^{7}$ It is interesting to note that phase changes are also thought to be a possible energy source for deep-focus terrestrial quakes (Kasahara 1981).

We think that phase transitions and internal magnetic fields are the most plausible sources of free energy in old neutron stars.

\section{DISCUSSION AND CONCLUSIONS}

It is perhaps no surprise that after surveying many aspects of the starquake model for $\gamma$-ray bursts, we have not reached any

\footnotetext{
${ }^{7}$ Bisnovatyi-Kogan and Chechetkin (1979) have emphasized that metastable nuclei are a source of free energy in neutron stars.
} 
firm conclusion regarding its validity. All that we can offer is an assessment of its strengths and weaknesses.

Starquakes have the following virtues. They can easily provide the energy necessary to power a single burst. They release energy in the low-entropy form of seismic waves which are transformed into Alfvén waves on time scales characteristic of observed $\gamma$-ray bursts. The energy density in the Alfven waves dominates the rest-mass energy density in the magnetosphere. The Alfvén waves may become nonlinear far from the stellar surface and accelerate the ambient plasma to energies that are limited by radiation reaction losses due to the emission of $\gamma$-rays. Thus, it is possible to imagine an efficient conversion of elastic energy to $\gamma$-ray energy.

Of course, the above scenario is replete with uncertainty. It is quite possible that plastic flow, and not brittle fracture, is the manner in which stress is relieved in neutron star crusts. The source of the free energy necessary to replenish the crustal stress in old neutron stars is not identified. The conversion of seismic waves into Alfvén waves, on the appropriate time scales and without the production of excessive crustal strains, requires that magnetic fields $\gtrsim 10^{11} \mathrm{G}$ cover at least part of the stellar surface. Such field strengths are larger than those gener- ally associated with old neutron stars; they certainly exceed the field strengths deduced from the rotational braking of millisecond pulsars. However, surface fields of $10^{11} \mathrm{G}$ may be small enough to switch off the pulsar mechanism. The propagation of Alfvén waves in low-density neutron star magnetospheres is poorly understood. The behavior of these waves and the manner in which they accelerate particles probably depend upon the degree to which the neutral plasma density exceeds the corotation charge density.

We close on an optimistic note. In spite of all the uncertainties that plague the starquake model, it appears to us to be the most viable model yet proposed for $\gamma$-ray bursts. Some of its difficulties may reflect our poor understanding of the magnetic and mechanical properties of neutron star interiors.

This research was supported by NASA grant NAGW 1303 and NSF grants AST86-15325 and AST86-1279. O. B. holds a Chaim Weizmann research fellowship at Caltech. We thank J. Arons, R. Jeanloz, D. Hartmann, E. S. Phinney, R. Romani, and $\mathrm{S}$. Woosley for informative discussions. We also thank the referee, R. Epstein, for helpful comments which improved a previous version of the paper.

\section{APPENDIX A}

\section{THE DISPERSION RELATION FOR RELATIVISTIC ALFVÉN WAVES}

We can understand the electrodynamic properties of a relativistic Alfvén wave by computing the dielectric response of the plasma. Since the electrons are mostly in their ground Landau levels, they form a one-dimensional plasma, undergoing $\boldsymbol{E} \times \boldsymbol{B}$ and polarization drifts perpendicular to the field and moving along the field in response to a field-parallel electric field. The dielectric tensor, evaluated in the center of momentum frame, is the same as that for a classical plasma with zero perpendicular temperature (e.g., Melrose 1980). The nonzero elements are

$$
\begin{aligned}
\epsilon_{x x} & =1+\frac{\omega_{P}^{2}}{\omega_{G}^{2}}\left\langle\gamma\left(1-\frac{k_{\|} v}{\omega}\right)^{2}\right\rangle, \\
\epsilon_{x z} & =\frac{\omega_{P}^{2}}{\omega_{G}^{2}} \frac{k_{\perp}}{\omega}\left\langle\gamma\left(1-\frac{k_{\|} v}{\omega}\right) v\right\rangle=\epsilon_{z x}, \\
\epsilon_{z z} & =1+\frac{\omega_{P}^{2}}{k_{\|}} \int_{L} \frac{d u \partial f / \partial u}{\omega-k_{\|} v}
\end{aligned}
$$

where the wave propagates in the $x-z$ plane at an angle $\psi$ to the magnetic field which lies along the $z$-axis. Here $\omega_{G}$ is the cyclotron frequency and $\omega_{P}=\left(4 \pi n e^{2} / m_{e}\right)^{1 / 2}$ is the formal plasma frequency for positrons and electrons combined. (The plasma is assumed to be charge-neutral. If this is not true, there will be additional contributions to the dielectric tensor.) Also, $f(u)$ is the one-dimensional particle distribution function evaluated at proper velocity $u=v\left(1-v^{2}\right)^{-1 / 2}$ and normalized to unity.

If most of the electrons have relativistic speeds, the dominant contributions to $\epsilon_{x x}, \epsilon_{x z}$, and $\epsilon_{z x}$ come from particles that are moving along the field, in the opposite direction to the wave. In this case we introduce a parameter

$$
\beta=\frac{2 \omega_{P}^{2}\langle\gamma\rangle}{\omega_{G}^{2}} \sim 10^{-5}\langle\gamma\rangle\left(\frac{n}{1 \mathrm{~cm}^{-3}}\right)\left(\frac{B}{1 \mathrm{G}}\right)^{-2},
$$

which is the ratio of the particle energy density to the magnetic energy density and is generally smaller than unity. Therefore,

$$
\epsilon_{x x} \simeq 1+\beta, \quad \epsilon_{x z}=\beta \tan \psi / 2 .
$$

By contrast, $\epsilon_{z z}$ is dominated by particles streaming along the field with the wave. Using the Plemelj relation in the usual manner, we obtain

$$
\epsilon_{z z}=1-\frac{\beta \omega_{G}^{2}}{\omega^{2}}+\frac{2 i \beta \omega_{G}^{2} \omega_{i}}{\omega^{3}}-\frac{\pi i \omega_{P}^{2}}{\omega^{2}}\left(\gamma^{3} \frac{\partial f}{\partial u}\right)_{u=\beta^{-1 / 2}},
$$

where $\omega_{i}$ is the imaginary part of the frequency responsible for wave damping. Particles with Lorentz factors $\gamma=\beta^{-1 / 2}$ Landau- 
damp the wave. Substitution into Maxwell's equations furnishes the dispersion relation

$$
\frac{c^{2} k_{\|}^{2}}{\omega^{2}}=1+\beta+\frac{\omega^{2} \tan ^{2} \psi}{\beta \omega_{G}^{2}},
$$

where we have only retained the leading terms, under the assumption that

$$
\omega \omega_{G} \ll \omega_{P}^{2}\langle\gamma\rangle \ll \omega_{G}^{2} .
$$

The damping decrement is given to leading order by

$$
\frac{\omega_{i}}{\omega}=\frac{\pi \omega^{2} \tan ^{2} \psi}{4 \omega_{G}^{2} \beta^{3 / 2}\langle\gamma\rangle}\left(u^{2} \frac{\partial f}{\partial u}\right)_{u=\beta^{-1 / 2}},
$$

and it is generally negligible.

The Alfvén waves therefore propagate without significant losses close to the star if they have linear amplitudes.

\section{APPENDIX B}

\section{COUPLING TO MAGNETOSONIC WAVES}

In $\S$ III we showed that seismic waves couple to fast magnetosonic waves only if $\theta_{i} \lesssim \tilde{v}_{s}\left(z_{\mathrm{ev}}\right) / c$. Because the horizontal wavenumber $k_{h}$ is related to the quantum number $l$ in a spherical star by $l(l+1)=k_{h}^{2} R^{2}$, this implies that substantial coupling occurs only for $l \lesssim \omega R / c$.

McDermott et al. (1984) have calculated the emission of electromagnetic waves produced by the oscillation modes of a neutron star in vacuum, assuming that the equilibrium dipole magnetic field has negligible effect on the mode frequencies and eigenfunctions. It is unlikely that real neutron stars would emit electromagnetic waves, because the magnetosphere plasma frequency greatly exceeds typical oscillation frequencies. However, fast magnetosonic waves have the same dispersion relation (36), so their emission is entirely analogous.

By use of the formalism from McDermott et al.(1984), it can be shown that the electromagnetic power radiated from the star is

$$
P=C(\omega, l) \frac{c}{8 \pi} B^{2} \eta^{2}(R) \frac{\omega^{2} R^{2}}{c^{2}},
$$

where $\eta$ is the characteristic magnitude of the surface displacement. We are interested in the regime $\omega R / c \gtrsim 1$, for which the coefficient $C(\omega, l)$ is of order unity for $l \lesssim \omega R / c$, and is given by

$$
C \sim \frac{l^{2}}{2 e}\left(\frac{\omega R e}{2 c l}\right)^{2 l}
$$

when $l \gtrsim \omega R / c$. The radiation of electromagnetic or equivalently fast magnetosonic modes is therefore negligible compared with the radiation of Alfvén modes.

\section{REFERENCES}

Alcock, C., and Illarionov, A. 1980, Ap. J., 235, 541.

Alpar, M. A., Anderson, P. W., Pines, D., and Shaham, J. 1984, Ap. J., 278, 791.

Baym, G., Pethick, C., and Sutherland, P. 1971, Ap. J., 170, 299.

Baym, G., and Pines, D. 1971, Ann. Phys., 66, 816.

Bisnovatyi-Kogan, G. S., and Chechetkin, V. M. 1979, Soviet Phys.-Uspekhi, 22,89.

Epstein, R. I. 1988, Phys. Rept., 163, 155.

Fabian, A. C., Icke, V., and Pringle, J. E. 1976, Ap. Space Sci., 42, 77.

Flowers, E., and Itoh, N. 1976, Ap. J., 206, 218.

Goldreich, P., and Julian, W. H. 1969, Ap. J. 157, 869

Hartmann, D., and Woosley, S. E. 1988, in Multiwavelength Astrophysics, ed.

F. Córdova (Cambridge: Cambridge University Press), chap. 10.

Illarionov, A., and Sunyaev, R. 1975, Astr. Ap., 39, 185.

Imamura, J. N., and Epstein, R. I. 1987, Ap. J., 313, 711

Itoh, N., Kohyama, Y., and Takeuchi, H. 1987, Ap. J., 317, 733.

Kasahara, K. 1981, Earthquake Mechanics (Cambridge: Cambridge University Press), pp. 106, 155.

Klebesadel, R. W., Strong, I. B., and Olson, R. A. 1973, Ap. J. (Letters), 182 L85.

Kouveliotou, C., Desai, U. D., Cline, T. L., Dennis, B. R., Fenimore, E. E. Klebesadel, R. W., and Laros, J. G. 1988, Ap. J. (Letters), 330, L101.

Laros, J. G., Evans, W. D., Fenimore, E. E., Klebesadel, R. W., Shulman, S., and Fritz, G. 1984, Ap. J., 286, 681.

O. Blaes, R. Blandford, P. Goldreich, and P. Madau: Theoretical Astrophysics 130-33, California Institute of Technology, Pasadena, CA 91125
Liang, E. P., and Petrosian, V. 1986, AIP Conf. Proc. 141, Gamma-Ray Bursts (New York: AIP).

Lyne, A. G., Manchester, R. N., and Taylor, J. H. 1985, M.N.R.A.S., 213, 613.

McDermott, P. N., Savedoff, M. P., Van Horn, H. M., Zweibel, E. G., and Hansen, C. J. 1984, Ap. J., 281, 746. Breach).

Minster, J. B. 1980, in Proc. Enrico Fermi Internat. School of Physics, Physics of the Earth's Interior, ed. A. Dziewonski and E. Boschi (New York: Academic).

Muslimov, A G and Tsygan A. I 1986, Ap. Space Sci., 120, 27

Pacini, F, and Ruderman, M. 1974, Nature, 251, 399.

Pines, D., and Alpar, M. A. 1985, Nature, 316, 27.

Schmidt, W. K. H. 1978, Nature, 271, 525.

Share, G. H., Matz, S. M., Messina, D. C., Nolan, P. L., Chupp, E. L., Forrest, D. J, and Cooper, J. F. 1986, Adv. Space Res., 6 (No. 4), 15.

Slattery, W., Doolen, G., and DeWitt, H. 1982, Phys. Rev. A, 26, 2255.

Sturrock, P. A. 1986, Nature, 321, 47.

Tsygan, A. I. 1975, Astr. Ap., 44, 21.

Weinberg, S. 1962, Phys. Rev., 126, 1899.
Melrose, D. B. 1980, Plasma Astrophysics, Vol. 1 (New York: Gordon \& 